\title{
Analysis of traffic safety for cyclists: the automatic detection of critical traffic situations for cyclists
}

\author{
S. Detzer, M. Junghans, K. Kozempel \& H. Saul \\ German Aerospace Center, Institute of Transportation Systems, \\ Traffic Management (DLR), Germany
}

\begin{abstract}
Over the past years, the bicycle has gained importance as a means of transportation in big cities. The use and acceptance of a bicycle as being an evolving means of transportation is highly linked to its transportation safety. Still, the risk of accidents is a dominant barrier. Even though the Federal Ministry of Transport, Building and Urban Development established a National Cycling Plan to enhance cycling and improve safety aspects, serious accidents still occur. Even if the number of traffic accidents is declining in Berlin, the consequences of bicycle accidents with physical injury are characterised by increasing results. Thus, it is proved that more than half of the accidents that involve bicyclists are caused by the cyclist itself. To understand causes of accidents and to eventually arrange preventive measures and enhance cyclists' safety, critical situations were detected. The application is based on cyclists' trajectories generated from video sequences. As a result, atypical and dangerous traffic situations can be identified automatically whereas rule violations can be detected manually. First experiences at an intersection in Berlin show a general applicability of this approach, which has to be widely tested at other intersections.
\end{abstract}

Keywords: traffic safety, traffic monitoring, bicycle traffic, camera recognition system, trajectories, situation detection. 


\section{Introduction}

Demographic change, rising bicycle participation and electric bicycles are developments that will affect the future of cycling. But how can cycling get safer?

The second most common accidents are those where bicyclists are involved after those with passengers in cars (2012: 19.3\%) [1].

In Berlin every third person injured in traffic is a cyclist [2]. Every two hours a bicycle accident happened in Berlin in 2012 (overall: 7.342 bicycle accidents) [3]. The most common reasons for cyclists' accidents are mistakes during a turn (2012: 53\%) and the disregard of the right of way (2012: 22\%). The main reasons for accidents of cyclists are the use of the wrong direction of the road (2012: 44\%) and violations when entering the flow of traffic (2012: 24\%) [3].

In this paper a system is introduced, which was developed in a project to find out more about the behaviour of cyclists in general and especially in their interaction with other road users at urban crossroads. For this, cyclists were detected and tracked at an intersection in Berlin with optical sensors. The resulting trajectories could then be classified and analysed according to anomaly and dangerousness, i.e. atypical and/or dangerous traffic behaviour.

The result of the investigation is a methodology that allows to analyse urban crossings and (accident black) spots and to identify measures to improve road safety, particularly for cyclists.

The paper is structured as follows: In section 2 the used method to detect and classify moving objects as well as the identification of atypical and dangerous situations is presented. Subsequently, the results of the detected trajectories are shown in regard to its classification, correctness and safety related parameters. Finally, a conclusion is given and an overview for further work is displayed.

\section{Methodology}

From a safety related point of view atypical and dangerous situations are of particular interest within the analysis of the behaviour of cyclists - both the behaviour of a single cyclist and in interaction with other road users. Such situations are an evidence of feasible conflict situations, which may occur frequently at an intersection. In addition, the consideration of safety related parameters and the analysis of traffic facilities can be carried out.

To look into this in more detail, an existing system for situation detection and assessment, which records and evaluates the interactions of cyclists at an urban intersection autonomously in reference to anomaly and dangerousness has been developed. These include the determination of safety related parameters (e.g. time to collision) and the frequency of red light violations.

The following method described includes the detection of cyclists by image processing and the identification of atypical and dangerous situations. 


\subsection{Image data processing}

The process used is divided into four steps: object segmentation, classification, tracking/prediction and classification of trajectories.

With optical sensors moving objects were detected in video images captured at the observed intersection (Rudower Chaussee/Wegedornstr). As a first step, the object segmentation is performed while the non-moving background is separated from the moving foreground. The detection of moving objects was performed by applying the Mixture of Gaussians background estimator.

For the determination and classification of the detected vehicles a Support Vector Machine (SVM) was implemented. It uses the shape of the convex hull of the traffic objects as feature vectors. The convex hull was equidistantly sampled and Fourier transformed. The coefficients of the transformed hull and the object size result together in the feature vector for classification.

Six possible object classes (cars, vans, lorries, buses, pedestrians and cyclists) were selected for the study (see Figure 1).
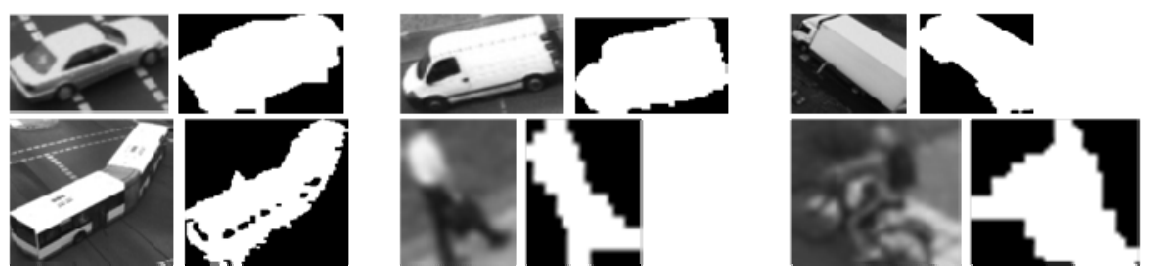

Figure 1: Typical shapes for cars, vans, lorries, buses, pedestrians and cyclists (from top left to bottom right).

With the help of a multi object tracking - Extended Kalman Filter - the disturbances of the measured values are filtered, the objects are tracked and their movements are predicted. This produces trajectories that have a scalable prediction horizon. These trajectories are the basis for the determination of atypical and dangerous traffic situations. In the following, the methods developed for the detection of atypical and dangerous situations are explained in detail.

\subsection{Identification of atypical situations}

Atypical situations refer to incidents, which differ from the usual case, but most of all present a danger to road users. Examples are U-turns, red light violations, sudden and hard braking or shorten the cycle path by crossing the roadway diagonally. In order to analyse these situations in more detail, a method for automatic detection in video recordings was selected and implemented, which is capable differentiating autonomously between typical and atypical situations without previous knowledge and allows to visualise and quantify selected atypical situations for a longer period. 
For the implementation the approach of Owens and Hunter [4] was applied. Advantage of this approach is that for the detection of atypical situations no fixed definition or modelling is required. Purely data-driven, the probability density function of the flow vectors and thus the motion is approximated.

The method adapts to the environment and learns normality from actual data from the video recordings. This is done by vector quantization using an artificial neural network: frequently occurring feature vectors are merged to "prototypes". Typical optical flow vectors consisting of position $(x, y)$ in world coordinates and speed $(d x, d y)$ are extended. The position, speed and acceleration are averaged by previous trajectory points, so that information about the moving direction can be encoded and the noise, in particular referring acceleration, can be reduced. Owens and Hunter use a sliding window denoted as function $s$ that is interpolating trajectory points to smooth the data but most notably to detect sudden directions changes in combination with splitting the components into the $x$ and $y$ direction. The resulting feature vector is as follows:

$$
F=\left(x y s(x) s(y) s(d x) s(d y) s\left(d^{2} x\right) s\left(d^{2} y\right)\right)^{T} .
$$

With the position $(x, y)$ and the derived values speed $(d x, d y)$, eqn (2), and acceleration $\left(d^{2} x, d^{2} y\right)$, eqn (3), - each separated in $x / y$ components of the Universal Transverse Mercator coordinate system is due to:

$$
\begin{gathered}
d x=x_{t}-x_{t-1} \\
d^{2} x=x_{t}-2 x_{t-1}+x_{t-2},
\end{gathered}
$$

and the smoothing function $s_{t}(x)$ as follows in eqn (4):

$$
s_{t}(x)=\mu\left(s_{t-1}(x)+(1-\mu)(x)\right) \text {. }
$$

Calculated feature vectors from the trajectories serve as input to a neural network. The artificial neural network used is a so-called Self Organising Map (SOM) or Kohonen map [5].

The SOM was trained with a total of 221 detected trajectories of bicyclists. In order to represent the trajectories appropriately and to reduce the loss of information, the SOM was built as a network of $20 \times 20$ neurons and trained with 10278 feature vectors from the 221 trajectories in 100,000 iterations. By means of the SOM a demonstration for regular or frequent movements for individual cyclists was created. With these movements, the trajectories of cyclists can be evaluated on other days.

20 trajectories of these 221 trajectories were classified as atypical. To be considered as atypical a trajectory must have been assessed with at least $90 \%$ as normal.

The analyses have shown that the classification in terms of anomaly enables autonomous evaluation of long periods and therefore a validation of the 
behaviour of cyclists at intersections is doable. The followed approach is capable and applicable in real-time without previous knowledge about the behaviour at an intersection or expected conflict situations. Furthermore, the algorithm is able to adapt to changing circumstances (e.g. traffic routing), so that atypical situations of cyclists (and other road users) can be identified very quickly.

\subsection{Identification of dangerous situations}

A dangerous or critical situation, in contrast to an atypical situation, is an event that may result in an accident. Here examples are driving against the direction of travel or driving with less safe distance and high speed.

Various traffic safety related parameters can be determined from the trajectories, which allow to identify and to predict critical situations $[6,7]$. In order to analyse these situations in more detail two well-known traffic safety related parameters were used for the analysis.

The first parameter is designated Time to Collision (TTC) an effective measure for rating the severity of conflicts [7]. That time which is expected for two objects at their present motion parameters until collision is going to happen [6]. For two successive vehicles the TTC is calculated by the distance between the two objects $(\Delta d)$ and divided by the speed difference of the two objects $(\Delta v)$ [8]. The equation is as follows:

$$
\mathrm{TTC}=\frac{\Delta \mathrm{x}}{\Delta \mathrm{v}}
$$

The detected trajectories of cyclists were filtered by a multi object tracker (Extended Kalman Filter) that predicts their positions in constant motion and thus it is possible to predict the times left over to collision, even when the conflict does not occur. The smaller the TTC, the more dangerous is the traffic situation. The analysis showed that critical situations or low TTC values are found frequently on the cycle path in west-east direction in interaction with vehicles, which are in the left turn manoeuvre (see Figure 2). Other clusters arise on the access roads from the south and west. Both cross points are typical accident situations for cyclists (see section 1).

The second variable examined is designated as Post-Encroachment-Time (PET) and describes the time difference that two objects "miss" each other in time. This means it is the time it takes until object 2 moves into this conflict area after object 1 left it $[6,9]$. The smaller this time, the more dangerous can the situation be. This parameter is interesting as soon as the lane of the two objects overlap. For the trajectories of bicyclists collected during the project, the PETs were determined with respect to all other road users. However, the parameter is not very meaningful in this case because for road users who follow others without collision PET values less than 4 seconds occur. The parameter is more significant considering only vehicles crossing each other in a minimal angle. 


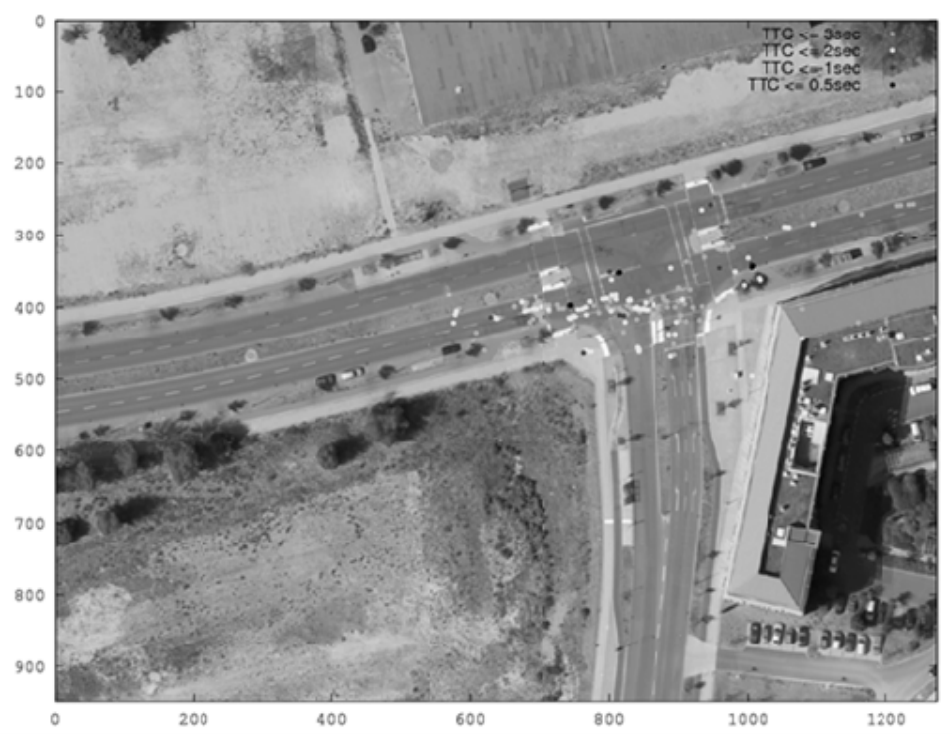

Figure 2: Distribution of TTC values.

\section{Data evaluation}

This section gives an overview about the observation area, the detected data and their results. For testing an intersection in Berlin was chosen, which is located close to the DLR and has a cycle path adjacent the road with an indirect cycling guide and separate bicycle traffic lights. Due to the separate and parallel cycle and pedestrian paths conflicts with pedestrians are rather unlikely. Cars and public transport have a separate parking area and cross the bike path only during the turn. Furthermore, three cameras are already installed, with a resolution of 1392 x 1040 pixels, which transmit 20 frames per second over gigabit ethernet to a video server, which is doing the image processing. Using these sensors, the traffic can be detected and examined at the intersection.

The aim was to get an overview of the behaviour of cyclists at this selected intersection on the basis of the developed system.

For this purpose, 353 cyclists were detected and analysed at seven different days and times during the period from July to October using the installed cameras.

\subsection{Classification of bicycles}

The traffic object recognition was evaluated with 60 minutes of imagery and 862 objects. This results in 534 cars, 101 vans, 151 lorries, 18 buses, 39 cyclists and 19 pedestrians. A total of $89 \%$ of the detected objects were correctly classified with the methodology used. On the left of figure 3 the number of correctly classified objects in relation to the actually occurred object is showed. By far the 
best recognition rates can be obtained for buses (100\%). $75 \%$ of the occurred cyclists were correctly classified. In contrast, problems for the detection of pedestrians occurred, only $39 \%$ were classified correctly. Almost all pedestrians classified as a bicycle, in particular when the pedestrian occurred in groups, which in turn lead to misclassification of them as cyclists. Overall, only $46 \%$ of detected cyclists are really bicycles (see Figure 3, right side). The main cause is the similar silhouette and size. To improve the classification accuracy other parameters, e.g. speed or position should be considered. These characters can be weighted according to credibility. In addition, the correctly identified motorcycles as two-wheeled, which so far are not explicitly distinguished from bicycles and thus need to be sorted manually.
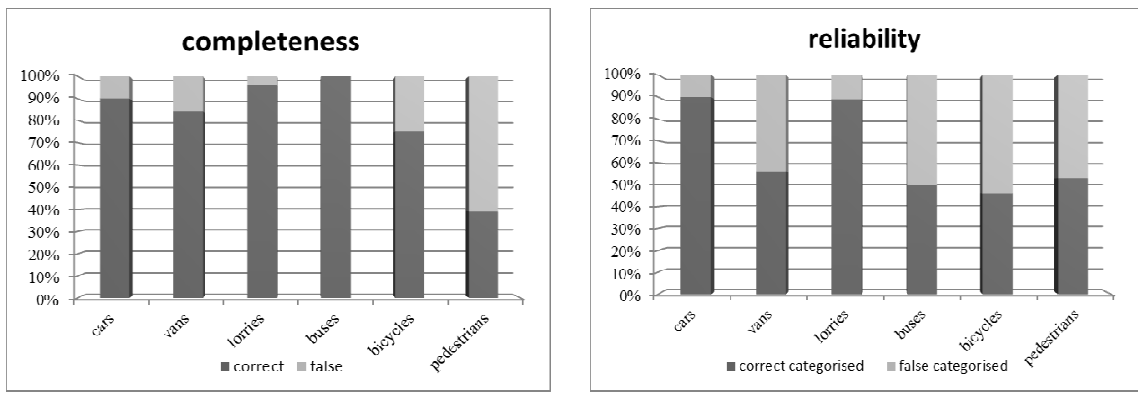

Figure 3: Recognition rate of individual classes (completeness) (left), recognition rate of individual classes (reliability) (right).

\subsection{Correctness of bicycle trajectories}

To check the accuracy of the bicycle trajectories a high-precision Trimble GPS receiver was used as reference. The GPS data were optimised in post-processing with the error correction data from the base station on the DLR buildings and have, as specified by the device, an accuracy of about 2-3 cm. As expected, more distant trajectories resulted in much larger disparities to GPS trajectory by projection errors. This is due to incorrect projections onto the road surface and because of the low detection accuracy by very low pixel resolutions of the objects. For instance, an image pixel has a size of almost 18 x $18 \mathrm{~cm}$ in $90 \mathrm{~m}$ distance. The method of the root mean square (RMS) was used for the determination of the error values. Trajectory positions with a distance between 22-72 meters from the camera have a RMS error of about 0.6-2.5 meters.

The most common sources of error for incorrect optical determination of position are:

- Glancing intersection: Its 3D-position is determined by the projection of the object balance point on the road surface. The further away an object is, the further the cut moves behind its actual position. 
- Occlusion: If an object is partially occlude by another object (moving or unmoved), the recognised shape and its center of gravity changes. Consequently, the position is projected incorrectly.

- Shade: When the shadow of an object is detected, the center of gravity is distorts, which yields to incorrect projection.

- Low contrast: Because of the low contrast to the background, the wheels of the bike occasionally are poorly or not recognised at all, thus the focus moves upwards and the projection is distorted. This occurs mainly on the road.

- Transparent wheels: As the wheels of most bicycles consist of spokes, at distant ones the road shines through, which makes them appear as background. This detection error shifts the objects centroid and causes incorrect projection.

\subsection{Determination of safety related parameters}

The collected bicycle trajectories were analysed and visualised with regard to their frequency and speeds. Figure 4 shows an accumulation of speed values between 11 to $18 \mathrm{~km} / \mathrm{h}$, but there is also a maximum value above $40 \mathrm{~km} / \mathrm{h}$ shown. The general average speed for cycling is between $10 \mathrm{~km} / \mathrm{h}$ to $20 \mathrm{~km} / \mathrm{h}$ [10]. As well the curves of the cyclist routes are shown in Figure 4. From this, the frequency of cycling routes on the cycle path can be identified, like in the image shown. In addition, violations of the rules are recognisable, e.g. driving across the intersection, no use of the bicycle path. For example, at left-hand side of the image (see figure 4) cyclists use the footpath to cross the intersection. The same applies to the top sideway.

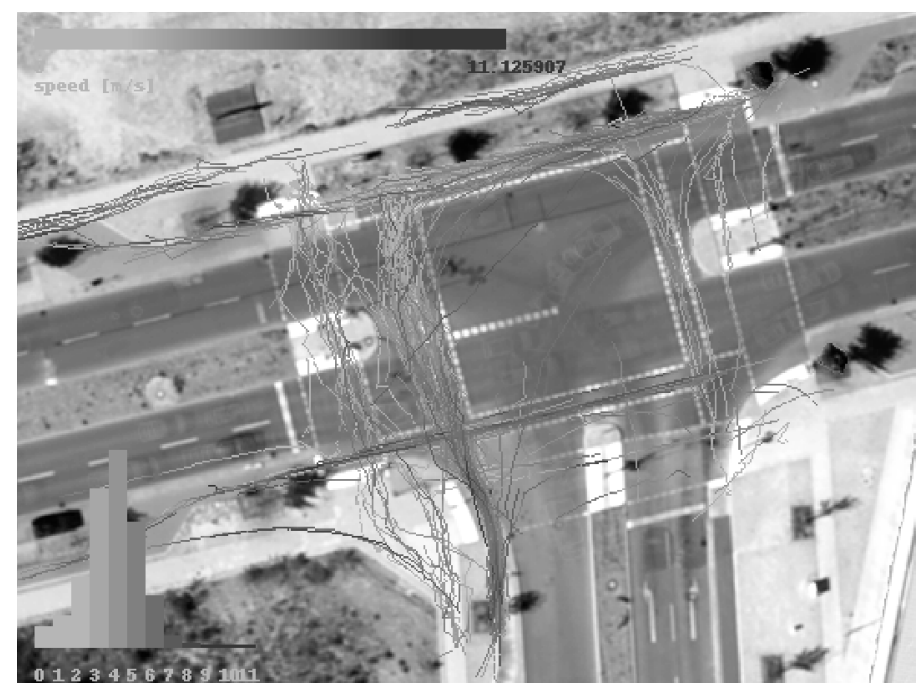

Figure 4: Velocities of cyclists. 
Unfortunately, the trajectories often tear off, because the wheels are hard to differentiate from the background. For tracking the objects appear too small and their shapes highly change. Furthermore occlusion prevents the monitoring of cyclists. For example are all bicycle trajectories torn on the sidewalk (center top). A tree occludes the view from the building.

The analyses of rule violations were based on a manual examination of the recorded videos of the bicycle trajectories at two days.

An automatic detection of red light violations was within the project due to lack of reliable data of traffic light circuits at the intersection not possible. Just a manual examination of the video recordings was realised.

Overall, the analysis showed that $65 \%$ of the investigated cyclists have acted compliantly. The following Figure 5 shows the irregular behaviour of cyclists.

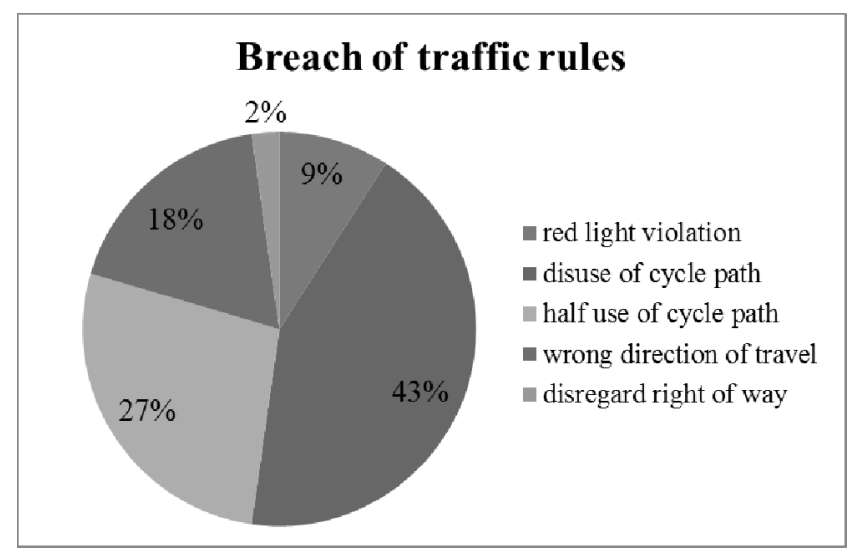

Figure 5: Overview of the type of rule violations.

The key violations are the disuse of the bike path (43\%) as well as the partly use of the bike path (27\%). Some cyclists used the sidewalk though the cycle path is located next to the sidewalk and not on the street (see also figure 4). The reasons for disuse are not clear. The cycle path was always free and was not blocked by any obstacle, such as construction sites, parking vehicles or resting persons. Partly at the beginning of the bicycle ride the sidewalk is used to cross the road and then the cyclists continue on the cycle path. Except of one cyclist who travels across the intersection, no one used the road during these two days. Another rule violation was the use of the wrong direction of travel. Within both days eight wrong-way drivers were recognised.

\section{Conclusion and suggestions for further work}

This project aimed to analyse the traffic safety situation for cyclists in Berlin. Therefore, a system for an automated detection of atypical and dangerous traffic situations with regard to the interactions of cyclists with other cyclists and traffic participants was developed and tested. The results showed that it is 
possible to analyse traffic areas with intersections to detect significant conflict points by an automated determination of traffic values and safety related parameters, see also the studies of Sakshaug et al. [11] and Ismail et al. [12]. It consists of a multi-camera system, which was mounted on a building at intersection Rudower Chaussee/Wegedornstraße in Berlin, Germany, and an image processing server that processes the images of three different views and provides traffic parameters and safety related parameters. The system provides traffic parameters (which were not the focus of the study) and safety related parameters in the case of near misses, accidents or atypical situations, e.g. U-turns, wavy lines. Critical and atypical situations could thus be automatically triggered and recorded for a post processing and manual evaluation, which was one the one hand done by a Self Organising Map (SOM) and on the other hand by safety related parameters like time to collision (TTC) and post encroachment time (PET). Currently, the developed system is able to inspect the results of the campaign "Radsicherheit in Berlin" (traffic safety for cyclists in Berlin) [13], see Figure 6.

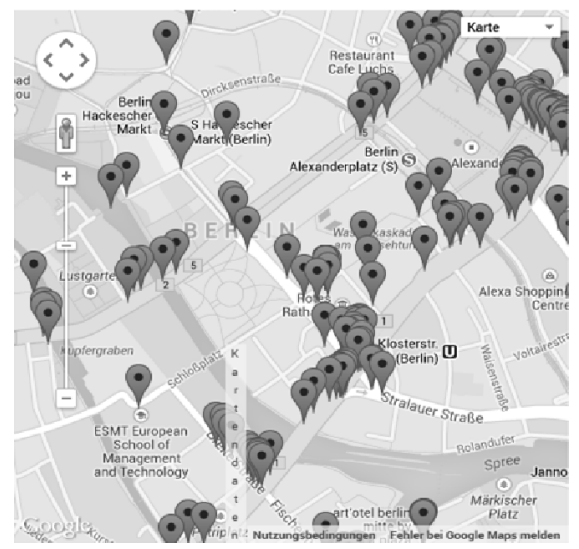

Figure 6: Snapshot of the website "Radsicherheit in Berlin", which shows conflict points reported subjectively by cyclists.

At this stage, objects that do not occlude each other can be detected with a precision of up to $89 \%$, correctly assigned to the class with up to $67 \%$ precision, followed across the entire detection area and predicted motions. An operation at night and under poor visibility and weather conditions is currently not sufficiently possible, but it will be realised by robust motion detection algorithms as shown in further developments of the well-known Lucas Kanade algorithm [14] or stabilising algorithms to avoid the tearing of trajectories. Furthermore, the method currently used for traffic participant classification of road users are to be improved and the motion model ought to be adapted, since the motion behaviour of pedestrians and possibly cyclists is completely different in comparison to motorised vehicles. For further investigations, the system needs to be transferred on our mobile Urban Traffic Research Car (UTRaCar), which is simply a VW T4 that is capable of processing the images of 3 cameras 
simultaneously. The UTRaCar has an extendable mast with a camera system and associated processing unit. It can monitor up to 12 hours of video with up to 20 images per second.

The question concerning traffic safety aspects of cyclists in Berlin cannot sufficiently be answered, since the results obtained show only very few information at the given intersection. Due to this fact, a longer test period, e.g. a month, will be necessary, to get more information and thus, it is aimed to observe black spots in Berlin, e.g. the intersections Ernst-Reuter-Platz, Moritzplatz or Kottbusser Tor, which suffer from up to 17 accidents involving cyclists in 2012. Furthermore, essential questions need to be answered concerning critical situations and accidents, concerning robust detection of objects and reliable Surrogate Safety Measures, for instance:

- Is there a (conditional) correlation between critical situations and accidents?

Under which circumstances and for which conflict and accident types can such correlations be proven? What kind of experiment can be realised to prove or even disprove it? Which methods are suitable for prove or disprove, e.g. Bayesian Networks (see Gregoriades and Mouskos [15])?

- Which Surrogate Safety Measures or which combinations of them instead of evaluating accidents and their severity need to be applied to identify the traffic safety risk at black spots?

- What do specific traffic parameters, e.g. traffic flow, density, in combination with Surrogate Safety measures mean for the traffic safety in intersections or road sections?

- Which other parameters, e.g. frequencies of hard braking, intensive and abrupt steering, can seem to be suitable for augmenting the data base for critical and accident situations? How can they be coupled with Surrogate Safety measures to describe the traffic safety risk?

- What do right of way and red light violations in general and in particular for cyclists say about traffic safety at intersections?

- How can we get the system robust against bad weather and illumination conditions?

For these reasons, it is intended to integrate more safety related parameters, which enables a significantly improved detection and prognosis of traffic conflicts. The parameters TTC and PET should be extended by the collision probability P (collision), see Saunier et al. [16], DRAC (deceleration to avoid accident) and DeltaV. The DRAC describes the braking acceleration, which is necessary to prevent an accident while DeltaV determines the severity of a conflict. Therefore it is supposed to allow statements about the severity of an upcoming accident. Another measurement is the detection of violations of the rights of way (e.g. red light violations). It is aimed to integrate the traffic light circuit plan or if not possible, to detect the phases on the basis of video detection. After the mentioned and other necessary extensions of the systems we will be able to analyse intersections with regard to safety aspects and recommend infrastructural and traffic control based measures in accordance with particularly German basic rules to improve the traffic safety of cyclists (and other traffic participants). 


\section{References}

[1] Statistisches Bundesamt Wiesebaden. Unfallentwicklung auf deutschen Strassen 2012, www.destatis.de, pp.25-26, 2013.

[2] Der Polizeipräsident in Berlin. Sonderuntersuchung "Radverkehrsunfälle" in Berlin 2012, http://www.berlin.de/polizei/verkehr/statistik.html, pp. 2, 2013.

[3] Der Polizeipräsident in Berlin. Verkehrssicherheitslage 2012 Berlin, Pressekonferenz am 06. März 2013, http://www.berlin.de/imperia/ $\mathrm{md} /$ content/polizei/strassenverkehr/unfaelle/statistik/verkehrssicherheitsla ge2012.pdf?start\&ts=1362472344\&file=ver-kehrssicherheitslage2012.pdf.

[4] Owens, J. \& Hunter, A., Application of the self-organizing map to trajectory classification, Proceedings of the Third IEEE International Workshop on Visual Surveillance (VS'2000), ser. VS'00. Washington, DC, USA: IEEE Computer Society, pp. 77, 2000.

[5] Kohonen, T., The self-organizing map, Proceedings of the IEEE, vol. 78, pp. 1464-1480, 1990.

[6] Trentacoste, M. F., Surrogate Safety Measures From Traffic Simulation Models, pp. 8-10, Virginia, 2003.

[7] Horst, A.R.A, Hogema, J.H., Time-to-Collision and collision avoidance systems, Proceedings of the 6th workshop of ICTCT, Salzburg, 1993.

[8] Freyer, J., Vernetzung von Fahrerassistenzsystemen zur Verbesserung des Spurwechselverhaltens von ACC, Audi Dissertationsreihe, Band 9, Cuvillier Verlag Göttingen, pp. 74-75, 2008.

[9] Horst, A.R.A. van der, A time-based analysis of road user behaviour in normal and critical encounters, $\mathrm{PhD}$ Thesis, Delft University of Technology, Delft, 1990.

[10] Radfahren macht Spass, http://www.radfahren-macht-spass.de/startseite/ wissenswertes/.

[11] Sakshaug, L., Laureshyn, A., Svensson, A., Hydén, C., Cyclists in roundabouts-Different design solutions, Accident Analysis and Prevention 42, pp. 1338-1351, 2010.

[12] Ismail, K., Sayed, T. and Saunier, N., Automated Analysis of Pedestrianvehicle Conflicts: Context For Before-and-after Studies, Transportation Research Record, Vol. 2198, pp. 52-64, 2010.

[13] Senatsverwaltung für Stadtentwicklung und Umwelt Berlin. Radsicherheit in Berlin Web Site, Berlin, https://radsicherheit.berlin.de/.

[14] Leich, A., Ein Beitrag zur Realisierung der videobasierten weiträumigen Verkehrsbeobachtung, Ph.D. Thesis, Dresden University of Technology, 2005.

[15] Gregoriades, A. \& Mouskos, K.C., Black spots identification through a Bayesian Networks quantification of accident risk index, Transportation Research Part C, Vol. 28, pp. 28-43, 2013.

[16] Saunier, N., Sayed, T., Ismail, K., Large Scale Automated Analysis of Vehicle Interactions and Collisions, Transportation Research Record, Vol. 2147, pp. 42-50, 2010. 